Courrier du Centre international Blaise Pascal

$34 \mid 2012$

Varia

\title{
Hommage à Jean Mesnard
}

\section{Maria Vita Romeo}

\section{OpenEdition}

Journals

Édition électronique

URL : http://journals.openedition.org/ccibp/291

DOI : 10.4000/ccibp.291

ISSN : 2493-7460

\section{Éditeur}

Centre international Blaise Pascal

\section{Édition imprimée}

Date de publication : 1 décembre 2012

Pagination : $37-41$

ISBN : 978-2-84516-612-7

ISSN : 0249-6674

Référence électronique

Maria Vita Romeo, «Hommage à Jean Mesnard», Courrier du Centre international Blaise Pascal [En ligne], 34 | 2012, mis en ligne le 03 décembre 2015, consulté le 15 septembre 2020. URL : http:// journals.openedition.org/ccibp/291

Ce document a été généré automatiquement le 15 septembre 2020

Centre international Blaise Pascal 


\title{
Hommage à Jean Mesnard
}

\author{
Maria Vita Romeo
}

1 Jeudi 19 janvier 2012, dans la salle grandiose du «Coro di Notte » du Monastère des Bénédictines, Université de Catane, il a été présenté le volume de Jean Mesnard, «Sui Pensieri di Pascal » (Éditeur Morcelliana, Brescia 2011), qui, grâce à l'éditrice Maria Vita Romeo (Université de Catane), offre la première traduction italienne d'une œuvre précieuse du grand pascalien français.

2 Une occasion très favorable donc, pour présenter au vaste public des spécialistes et des étudiants de l'Université de Catane aussi bien la traduction italienne d'un livre, qui est universellement reconnu comme un classique dans la littérature critique sur Pascal, que son illustre auteur, membre de l'académie des Sciences Morales et Politiques de France. En vérité, la présentation de Jean Mesnard à Catane ne serait pas un véritable scoop, parce que depuis une dizaine d'années le grand Académicien fait partie de la famille de l'Université de Catane. Et demeurent mémorables, par exemple, la dévotion et l'affection que lui donnent ponctuellement les jeunes de l'Université de Catane, chaque fois que la chaire de Philosophie Morale leur présente l'occasion de l'écouter pendant un colloque ou un séminaire.

3 C'est pour cela que les organisateurs ont pensé cet événement soit comme une présentation du volume, soit comme un véritable "Hommage à Mesnard", donc comme une occasion de réflexion rigoureuse et d'affectueuse amitié en l'honneur de Jean Mesnard et de son travail. Pour ce faire, coordonnés par Giuseppe Pezzino (Université de Catane), deux émérites spécialistes de la pensée philosophique du XVII ${ }^{e}$ siècle ont présenté ce volume: Giuseppe Bentivegna (Université de Catane) et Domenico Bosco (Université Catholique de Milan). Ceux-ci ont analysé en profondeur aussi bien la pensée de Pascal que les caractères spécifiques de la personnalité scientifique de Mesnard.

Dans la communication intitulée «I Pensieri di Pascal nella interpretazione di Jean Mesnard », Giuseppe Bentivegna a accompagné les auditeurs dans l'univers de Mesnard par l'analyse du livre : « un livre qui ne peut pas dormir sur les étagères poussiéreuses d'une bibliothèque, car il s'agit d'un texte vif, un texte qui discute, parfois aussi minutieusement, les plus influentes thèses interprétatives de la figure et de l'œuvre de 
Pascal ». Selon Bentivegna, Mesnard suit une ligne d'exposition et d'argumentation qui présente au lecteur un Pascal original, qui, comme ses Pensées, trouve toujours quelque chose à dire à l'homme de toute époque. Le message de Mesnard est clair : on ne peut pas parler absolument d'un Pascal dimidiatus, divisé en deux, qui avant opte pour la science et puis l'abandonne en faveur de la foi religieuse, mais d'un Pascal unitaire, savant et mystique, philosophe et polémiste, homme de la raison et homme du cœur. «Dans les mots de Mesnard - selon Bentivegna - la thèse de la continuité pascalienne entre savant et mystique est confirmée ; il s'agit d'une thèse qui répugne à n'importe quelle interprétation romantique, qui superpose et presque annule la tension unitaire des profondes recherches physique-mathématiques et religieuses, en vidant la tension spéculative et affective en lien indissoluble, à l'avantage d'un sentiment esthétique de la vie et de Dieu ».

La communication de Domenico Bosco, intitulée «Il Pascal di Jean Mesnard. Un Pascal "fedele... per tutti" ", présente au lecteur tout de suite celui qui à bon droit est considéré comme le plus grand maitre des études pascaliennes aujourd'hui. Le mérite de Mesnard, affirme justement Bosco, est de nous avoir légué un Pascal «intégral », un "Pascal ronde-bosse ", qui naît de la recherche minutieuse et soignée du philologue, lettré, historien, théologien et philosophe; Mesnard a su unifier le Pascal aux mille aspects, en dirigeant et en orientant aussi les recherches de savants tous différents entre eux et qui, aujourd'hui, ont fini par figurer parmi les spécialistes les plus éminents de Pascal.

6 Ainsi, quiconque s'approche de la «planète Pascal» ne peut pas éviter de faire ses comptes avec Mesnard et avec sa riche production scientifique, (on songe à l'édition critique monumentale des Cuvres complètes, avec le riche apparat de notes et textes, qui placent Pascal dans son contexte plus propre) où érudition et idée, idée et fait tendent à se marier, en devenant un aide "pour une compréhension plus adéquate de l'unique Pascal existant, cet homme du dix-septième siècle qui vit dans un réseau de relations, qui s'exprime dans les débats scientifiques de l'âge classique; qui, en rencontrant la "religion" de Port-Royal, toute imprégnée de l'esprit d'Augustin, se consacre à une apologie du christianisme ».

7 Dans son intervention Maria Vita Romeo n'a certainement pas caché l'émotion mêlée d'un grain d'orgueil pour avoir eu l'honneur de produire la première traduction italienne de l'œuvre de Monsieur Jean Mesnard, « Sui Pensieri di Pascal », qui représente un point fixe, pour utiliser un thermes cher à Pascal, soit pour les spécialistes soit pour ceux qui s'approchent pour la première fois de la pensée du grand Clermontois.

En effet, avec cette entreprise l'éditrice a parié non seulement avec soi-même, mais surtout avec tous ceux qui ont cru en elle : en tout premier l'auteur; et puis professeurs illustres - que Maria Vita Romeo a publiquement remerciés - comme Domenico Bosco de l'Université Catholique de Milan, Dominique Descotes de l'Université "Blaise Pascal" de Clermont-Ferrand, Gérard Ferreyrolles de l'Université Paris - Sorbonne, Philippe Sellier de l'Université Paris - Sorbonne et, last but not least, Giuseppe Pezzino de l'Université de Catane.

9 Selon Maria Vita Romeo, la traduction de ce livre ne pouvait plus être renvoyée surtout dans ce panorama culturel italien, où l'on assiste, depuis d'une décennie, à un renouveau vif et fécond des études pascaliennes et où la chaire de Philosophie Morale de l'Université de Catane constitue un des centres de la recherche scientifique pascalienne. 
10 Maria Vita Romeo souligne en outre que Mesnard, par sa riche production scientifique, brillamment synthétisée dans ce livre, a mis fin à une série de préjugés sur Pascal, en se confrontant avec trois précédentes lignes d'interprétation : la lecture voltairienne du dix-huitième siècle, qui décrit Pascal comme un misanthrope sublime; l'image romantique du dix-neuvième siècle d'un Pascal angoissé, qui cherche dans la foi un refuge pour son désespoir; et finalement l'image du vingtième siècle, proposée par Lucien Goldmann, d'un Pascal porteur d'une image tragique. Voilà pourquoi ce livre de Mesnard, pour la clarté et la rigueur des idées et pour la richesse des renseignements, constitue incontestablement un précieux instrument de travail soit pour naviguer mieux dans l'« univers Pascal ", soit pour comprendre la personnalité scientifique de Mesnard qui se révèle non seulement un historien remarquable de la littérature, mais aussi un excellent spécialiste qui procède avec sûreté sur le terrain de la philosophie, de la science, de la théologie, de l'esthétique et de la psychologie.

11 Le discours de clôture de Jean Mesnard a couronné une journée riche d'érudition et d'amitié, en donnant des moments d'émotion profonde quand il a parlé de l'affection qui le lie à Catane, où il a trouvé non seulement un cadre idéal pour la recherche et le savoir, mais aussi un haut lieu d'émotion. Le nom de Catane est évocateur de moments riches selon les dires mêmes de Jean Mesnard qui a rappelé sa première arrivée dans la ville de l'Etna à l'occasion des Journées Pascal 2004, organisées par Giuseppe Pezzino et Maria Vita Romeo, auxquelles ont succédé une série de rencontres culminant dans le «Colloque Port-Royal et la philosophie » de 2010.

12 À cette série d'émotions - affirme Mesnard - s'ajoute l'émotion qu'il a éprouvée de voir la traduction italienne d'un de ses « livres les plus importants et les plus difficiles », Les Pensées de Pascal ; un livre « dont l'histoire est aussi mon histoire »! Et ce n'est pas un pur hasard que Jean Mesnard a intitulé sa communication: «Les livres et la vie». En effet cet ouvrage, Les Pensées de Pascal, qui a vu la lumière dans la première édition de 1976, est avant tout un bilan des recherches de Jean Mesnard, culminant dans l'édition de 1993, enrichie de documents et d'une réflexion critique plus soignée. Cette dernière édition naît grâce à " une multitudes de retouches et d'additions qui, sans donner naissance à un ouvrage très différent, ouvrent la voie à de nouvelles orientations de lecture ", grâce aussi à des recherches nées par un groupe d'élèves de Jean Mesnard et de spécialistes éminents comme Pol Ernst, Michel Serres, Louis Marin, Dominique Descotes, Pierre Force et Laurent Thirouin. De là une vraie lectio magistralis sur Pascal, pour la compréhension duquel «il faut partir du langage même de Pascal», « de sa culture, laquelle accordait une place exceptionnelle aux mathématiques ». Et en effets, «l'œuvre scientifique est la seule susceptible de conduire à la profondeur, car l'esprit de Pascal à l'été intimement formé par les sciences ».

13 Ces deux dernières interventions - celle de l'auteur et celle de l'éditrice de la traduction italienne - ont sans aucun doute offert à la journée d'étude quelques notes personnelles d'expériences de travail, quelques fragments d'un vécu laborieux, lesquels ont souligné ou dévoilé des aspects inconnus du travail fatigant et exaltant qui a porté d'abord à la réalisation du livre et puis à sa traduction.

14 À vrai dire, cet « Hommage à Jean Mesnard » a aussi renfermé un sentiment de fête et un grain d'orgueil légitime parce que, avec la traduction de ce livre important - pour la noble maison d'édition Morcelliana de Brescia-le Centre d'études pascaliens de Catane, qui gravite autour de la chaire de Philosophie Morale et de celle de Philosophie 
Éthico-politique, a conquis un autre objectif après celui-là de la réalisation à Catane du Colloque International « Port-Royal et la philosophie » (8-10 novembre 2010).

On peut dire aussi que cet « Hommage » va se joindre idéalement aux deux célébrations $\mathrm{du} 90^{\mathrm{e}}$ anniversaire de Jean Mesnard qui se sont déroulées d'abord à Catane et puis à Paris. Cet « Hommage » donc constitue aussi un cadeau pour « le Maître », qui sait être aussi rigoureux dans les études (sa sévérité est légendaire!), qu'affectueux dans l'amitié. Un cadeau pour Jean Mesnard, qui offre un inimitable exemple d'activité scientifique incessante et riche, et qui nous a appris que «Pascal sert à penser ». Une leçon qui vaut autant dans la recherche que dans la vie. En effet - comme on lit dans une table en bois gardée par l'un de ses élèves les plus chers - « Un Pascalisant ne peut pas être foncièrement mauvais »!

En conclusion il faut souligner que cette journée mémorable s'est terminée avec une expression chorale de gratitude pour Monsieur Mesnard et son école, laquelle fusionne harmonieusement la rigueur scientifique et la chaleur humaine, une école qui continue à s'agrandir et à former de nouveaux élèves parmi lesquels osent espérer d'être inclus les chercheurs de Catane. 